\title{
Basidiomicetes de Costa Rica. Nuevas especies de Exobasidium (Exobasidiaceae) y registros de Cryptobasidiales
}

\author{
Luis D. Gómez P. ${ }^{1}$ y Liuba Kisimova- Horovitz ${ }^{2}$ \\ 1 Academia Nacional de Ciencias, Apartado 676-2050, Costa Rica, ldgomez@hortus.ots.ac.cr \\ 2 Spezielle Botanik Mykologie, Universität Tübingen, Alemania.
}

Recibido 19-I-1998. Corregido 24-VIII-1998. Aceptado 17-IX-1998.

\begin{abstract}
Six new species in the genus Exobasidium are described: E. aequatorianum n. sp., parasitic on Vaccinium crenatum (Don) Sleumer from Ecuador where it is widely distributed; E. arctostaphyli Harkn., found on Arctostaphylos arbutoides (Lindl.) Hemsl., and on Comarostaphylos costaricensis Small in Costa Rica is redescribed; E. jamaicense n. sp., on Lyonia jamaicensis (Swartz) D. Don from Jamaica and possibly throughout the Caribbean range of the host genus; E. disterigmicola n.sp., on Disterigma humboldtii (Kl.) Nied., from the Talamanca Range, Costa Rica and possibly, throughout the range of its host, E. sphyrospermii $n$. sp.,on Sphyrospermum cordifolium Bentham in Costa Rica, E. poasanum n. sp., on Cavendishia bracteata (R. \& P. ex J. St.-Hil.) Hoer., from the Poás massif in Costa Rica. Exobasidium escalloniae Gómez \& Kisimova, described from Costa Rica, is now known to occur in Ecuador on the same host, Escallonia myrtilloides L.f. Exobasidium, vaccinii (Fkl.) Wor. is here reported from Vaccinium floribundum H.B.K. from various Ecuadorean localities, and E. pernettyae n. $s p$. is described as a parasite of Pernettya prostrata (Cav.) DC in Costa Rica. With the exception of Escallonia, of saxifragaceous affinities, all hosts belong in the Ericaceae. Exobasidium arctostaphyli is a first record for the neotropical region. With the exception of $E$. jamaicense, not seen in the wild by us, all others have been found associated with small slugs (Mollusca: Limacidae) which are suspected to be one of the vectors of the parasite. Some biogeographical considerations are presented. The Cryptobasidiaceae Clinoconidium bullatum Syd., not collected since first described in 1925, and Clinoconidium farinosum (P. Henn.) Pat., are both parasites of Lauraceae, Cinnamomum sp. and Ocotea monteverdensis Burger, respectively, and were found in the Monteverde Reserve, Costa Rica. Drepanoconis larviformis (Speg.) Speg. is reported as a parasite of Aiouea costaricensis (Mez) Kosterm. from the cloud forests of San Gerardo de Dota, Costa Rica. Clinoconidium farinosum and Drepanoconis larviformis constitute new records for Central America.
\end{abstract}

Key words: Exobasidium, Clinoconidium, Drepanoconis, Ericaceae, Grossulariaceae, Saxifragaceae, Lauraceae, Cryptobasidiaceae, Exobasidiaceae, basidiomycetes, neotropical, phytopathology, Costa Rica, Ecuador, Limacidae, Mollusca, mycogeography, fungi.

Desde nuestra primera publicación sobre Exobasidiales (Gómez \& Kisimova 1997), hemos tenido la oportunidad de estudiar una amplia serie de materiales neotropicales que amplían la distribución geográfica y duplican el número de especies de Exobasidium conocidas en la región mesoamericana, el Caribe y los Andes ecuatorianos. De material procedente de la Reserva Forestal de Monteverde se registran una nueva localidad y una especie de Clinoco- nidium, y del bosque nublado de San Gerardo de Dota se comunica la presencia de Drepanoconis, ambos Cryptobasidiales son nuevos para el área.

\section{MATERIALES Y MÉTODOS}

Los mismos métodos descritos en Gómez \& Kisimova (1997). Los ejemplares se depositan 
en el Herbario de la Universidad de Costa Rica (USJ), el Herbario del Museo Nacional de Ciencias Naturales, Quito, Ecuador (QCNE), Jardín Botánico de Misurí, E.E.U.U. (MO), y Herbario, Universidad de las Indias Occidentales, Jamaica (UCWI).

Exobasidium aequatorianum Gómez \& Kisimova, nov. sp.

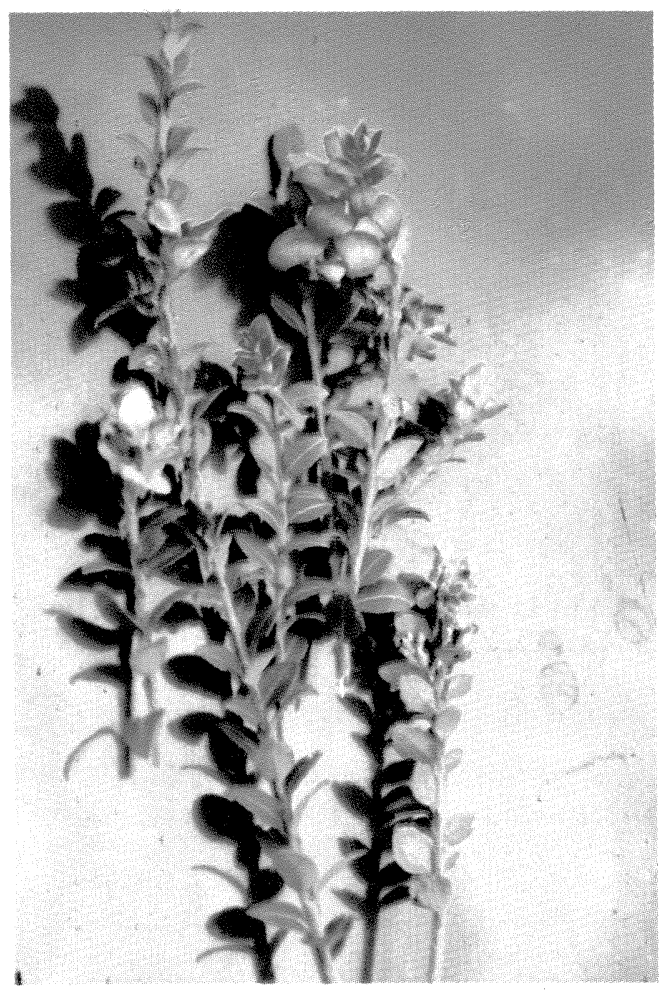

Fig. 1. Exobasidium aequatorianum infectando talluelos de Vaccinium crenatum, holotipo, Cotopaxi, Ecuador. Foto: Luis D. Gómez (L. D. G.)

Parasiticum, resupinatum, effusum, hypogenum. Systema hyphale monomiticum, hyphis 1.2 - 2.3(2.5) $\mu$ m latis, efibu-latis, tenuitunicatis, hyalinis, pauce septatis. Basidia cylindrico-clavata, 13 - $22 \times 6.3$ - $7.4 \mu \mathrm{m}$, 2-sterigmata (raro 3-sterigmata), sporis ellipsoideis $12 x$ $4 \mu m$, unisep-tatis, hyalinis, tenuitunicatis. Conidia, ut videtur, nulla.

Holotypus: En Vaccinium crenatum (Don) Sleumer, creciendo en matorrales del páramo asociado con Chuquiraga jussieui, Werneria crassifolia, Diplostephium sp.(Asteraceae), Gentianella cerastoides, Halenia sp. (Gentianaceae), Brachyotum ledifolium (Melastomaceae) y Stipa ichu (Poaceae). Ecuador, Volcán Cotopaxi, Provincia Cotopaxi, 3500 m, Gómez \& Hewson 25964, 29 octubre 1997 (QCNE). Isotypus: USJ. Paratypi: loc. dat. cit. Gómez \& Hewson 25966, 25970 (USJ, QCNE).

Materiales adicionales: Ecuador: Prov. Cotopaxi: Faldas SE del Coto-paxi, aprox. 10 km NE de Latacunga, en plantación de coníferas micorrízicas con Suillus luteus, $3300 \mathrm{~m}$. Gómez \& Hewson 25972, 29/10/97 (USJ); Borde de carretera, Páramo de Millán, 3000 m, Gómez \& Hewson 25973, 29/10/97 (USJ). Provincia Tungurahua. Estribaciones occidentales del Tungurahua, 3350 m, Gómez \& Hewson 25975, 29/10/97 (QCNE, USJ). Provincia Imbabura. Páramo El Angel, 3200 m, asociado con Espeletia hartwegiana, Hypericum laricifolium, Vaccinium floribundum. Gómez \& Hewson 25967, 25969, 03/11/97 (QCNE, USJ).

Hongo parásito en Vaccinium crenatum (Don) Sleumer, hipófilo, que cubre todo el envés de las hojas. Micelio intercelular, de hifas monomíticas, hialinas, de 1.2 - 2.3(2.5) $\mu \mathrm{m}$ de diámetro, relativamente uniformes, de paredes delgadas, con pocos septos dispersos. Himenio efuso en envés foliar. Basidios cilíndrico-clavados, 13 - $22 \times 6.3$ - $7.4 \mu \mathrm{m}$, hialinos, con 2 esterigmas (en unos pocos basidios se observaron hasta 3 esterigmas) vermiformes, de 3 - 4 $\mu \mathrm{m}$ de largo. Esporas característicamente curvadas hacia la base, elipsoidales, 12 - $4 \mu \mathrm{m}$, hialinas, de paredes delgadas. Conidios (blastoconidios) aparentemente ausentes.

El hongo causa una hipertrofia de los talluelos de $4^{\circ}$ y $5^{\circ}$ orden o retoños anuales con las siguientes características: 1- engrosamiento uniforme, no fusiforme, del eje del talluelo, con crecimiento anormal en tamaño y densidad de los pelos superficiales y una alta concentración de pigmentos rojos. El talluelo infectado es muy frágil y quebradizo. 2- Agrandamiento de las hojas del eje parasitado. El aumento de área foliar es del orden de $30-50 \%$, las clorofilas son 
reemplazadas por antocianinas, la haz es de un color rojo carmesí brillante. Las venas foliares, immersas en hojas no parasitadas, con la infección son visibles en gran detalle. Hay una disminución en la longitud de los entrenudos por lo que la hipertrofia en pleno desarrollo asemeja una "flor" terminal en el talluelo.

La nueva especie es, en términos generales, afín con Exobasidium vaccinii (Fkl.) Woronin, del que difiere por las dimensiones de basidios y esporas, y con E. talamancense Gómez \& Kisimova por el tipo de lesión que forma aunque en esta última los brotes infectados se producen en los tallos primarios, con transformación importante del leño y la hipertrofia presenta elongación de entrenudos y un gigantismo en las hojas infectadas, además de diferir de E. aequatorianum por las dimensiones de sus basidios y esporas.

Exobasidium arctostaphyli Harkn., California Acad. Sci. 1: 30. 1886. (= E. vaccinii var. arctostaphyli (Harkn.) Savile) (Figs. 2, 9).

Parásito que causa "agallas coraloides" en troncos vetustos además de hipertrofias en yemas vegetativas terminales, caracterizadas por abultamiento fusiforme del tallo, pérdida de clorofilas que son sustituidas por antocianinas y carotenoides, inhibición del desarrollo de la lámina foliar. El himenio es escaso, con afloración de basidios dispersos, sin formar una "felpa" evidente o sustancial. Basidios mazudos, 28 - 32 × 6 - $7 \mu \mathrm{m}$ 4-esterigmas, los esterigmas gruesos, digitiformes. Esporas de 6 - 12(13.5) x $2.5-3.5(4) \mu \mathrm{m}$. Las esporas son elipsoidales $y$ redondeadas en los extremos, cuando maduran tienden a aglutinarse en tetradas o triadas tanto en el basidio como aisladas de el y forman artrosporas de paredes gruesas y biseptadas. El micelio intercelular es profusamente septado y los probasidios son globoso-piriformes, de dimensiones diversas.

Material estudiado: Costa Rica: En arbustos de Arctostaphylos arbutoides ( Lindl.) Hemsl., Restaurante Las Torres, Cerro Buenavista, 3000 m, Prov. San José, 19/12/1997, L.D.Gómez 25984 (USJ); Cerro Buenavista, 1 $\mathrm{km}$ al NO de tajo al pie de torre repetidora,

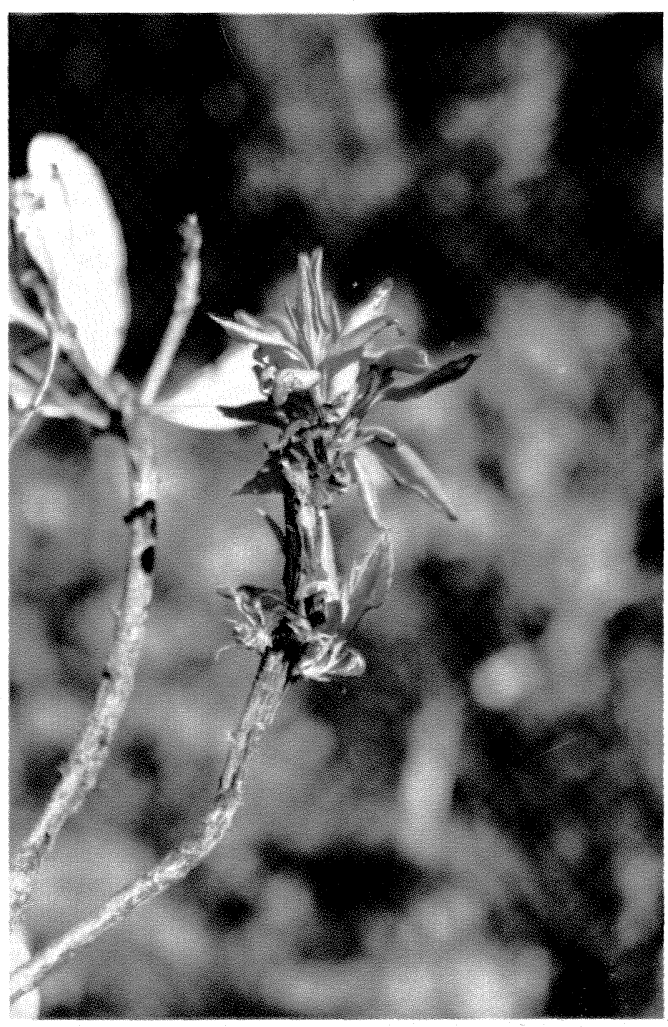

Fig. 2. Exobasidium arctostapyli. Hipertrofia de yema terminal de Comarostahlos costaricensis. Foto: L. D. G.

Prov. San José, 3100 m, 19/12/1997, L.D. Gómez 25985 (USJ); $2 \mathrm{~km}$ al O de intersección de Carretera Interamerica Sur con camino a San Gerardo de Dota, 2800 m, Prov. San José, 19/12/1997, L. D. Gómez 25986; Sobre Comarostaphylos costaricensis Small, $(=$ A. arbutoides v. costaricensis (Small) Wilbur \& Luteyn) Antiguo cráter Reventado, Volcán Irazú, 2700 m, Prov. Cartago, 20/12/1997, L. D. Gómez \& I. Chacón 25988 (USJ); loc. cit., Gómez \& Chacón 25989 (USJ); loc. cit., Gómez \& Chacón 25991 (USJ); La Alejandrina, km 93 Carretera Interam. Sur, Prov. San José, 2800 m, 20/12/1997, L. D. Gómez 26004 (USJ).

El género Arctostaphylos Adans., es parasitado por Exobasidium uva-ursi (Maire) Juel (= E. karsteni Sacc. \& Trott. f. uva-ursi (Maire) Sacc. \& Trott.) y por E. vaccini-uliginosi Boud. in Boud. \& Fisch., en la especie paleártica hospedera. En América del Norte se comunica de varias especies de Arctostaphylos 
(Cash 1953; Cooke 1955; Shaw 1973). Aunque el parásito encontrado en Costa Rica se encuentra sobre el segregado Comarostaphylos Zucc., asignamos la especie del hongo al concepto propuesto por Harkness (loc. cit.), al que agregamos detalles del micelio y de las esporas aglutinantes y formacion de artrosporas biseptadas. Consideramos esta especie distinta de $E$. vaccinii Wor. sensu Savile (1959).

Exobasidium disterigmicola Gómez \& Kisimova, sp. nov. (Fig. 3).

Parasiticus in foliis caulibusque Disterigmae ericacearum proliferationis scopariaeformis formans. Mycelium ligno systemicum. Systema hyphalis monomiticus textura intercellulis praecipue intricata interdum subporrecta, hyphis efibulatis, cylindraceis, tenuitu-nicatis, 1.2 - $3 \mu \mathrm{m}$ crassis, pallidissime ambarinis, plus minusve regulariter septatis. Hymenium folio effusum, hypophyllum. Basidia ut videtur

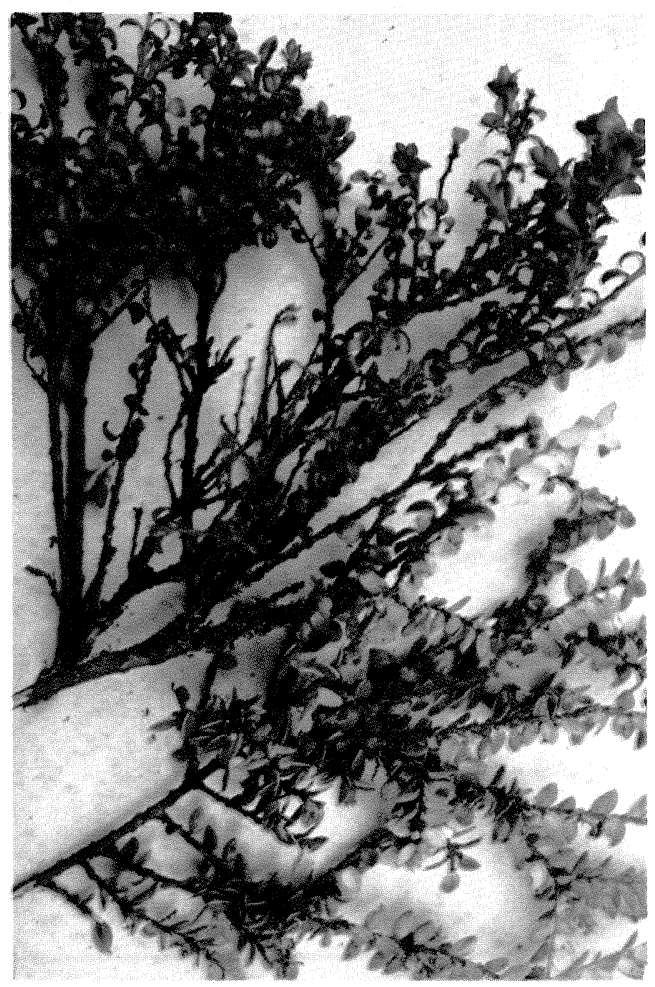

Fig. 3. Exobaidium disterigmicola. "Escoba de bruja" en Disterigma humboldtii, holotipo, Volcán Poás, Costa Rica. Foto: L. D. G. nulla. Conidia $12-15.5 \times 2.8-3.2 \mu \mathrm{m}$, lacteoso-hyalina.

Holotypus: Costa Rica: En Disterigma humboldtii (Kl.) Nied., Prov. de Cartago, entrada a torre 34 de ICE, Interamericana Sur, km. 70, 2735 m, L. D. Gómez 25958, 4/10/97 (USJ). Paratypus: loc. cit., L. D. Gómez 25959 (USJ). Otros materiales: En bosque que rodea la Laguna Botos, Volcán Poás, 1600 m, Prov. Alajuela, L. D. Gómez \& I. Chacón 25980, 15/10/97 (USJ); km 72 Interamericana Sur, Prov. Cartago, 2700 m, 20/12/1997, L. D. Gómez 26005 (USJ).

Hongo endógeno parásito de Disterigma humboldtii (Kl.) Niedenzu. Micelio sistémico en la corteza del leño, con un sistema vegetativo de hifas monomíticas que se extienden por entre las células con una textura entretejida aunque en algunas superficies parietales celulares se alinean en forma porrecta, sin fíbulas, de paredes delgadas, cilíndricas, regulares, de 1.2 - $3 \mu \mathrm{m}$ de diámetro, ambarino muy pálido y con septos simples más o menos regularmente distribuidos. El himenio aflora en toda la superficie del envés de las hojas del tallo infectado, forma una capa afelpada de hifas inicialmente reptantes y en sus ápices erectas. No se observaron basidios o basidiosporas pero sí numerosos conidios de $12-15.5$ x $2.8-3.2$ $\mu \mathrm{m}$, lacteoso-hialinos.

El parasitismo induce una proliferación multiramosa, ("escoba de bruja") con ramillas delgadas, enrojecidas, enclenques y quebradizas, muy numerosas. En la mitad distal de las ramillas las hojas enrojecidas en la haz, el envés totalmente ocupado por las hifas conidiógenas. Las hojas no sufren deformación y se diferencian en sus dimensiones, más pequeñas, de las hojas no infectadas.

Difiere de las especies ericófilas de Exobasidium por la formación de "escobas de bruja" en los tallos y por la aparente reproducción exclusiva por conidios. Por no causar hipertrofia foliar en los tallos infectados, se asemeja a E. vaccinii-uliginosi Boud. in Boud. \& Fisch. (Soc. Bot. France 41: CCXLIV. 1894) pero esa especie no produce escobas o conidios. Hasta la fecha, la única proliferación en escoba en 
ericáceas es la que produce Pucciniastrum goeppertianum (Kuhn) Kleb, Uredinales, Pucciniastraceae, en Vaccinium spp. y en Thibaudia spp. (Hiratsuka 1958, Mulenko 1994, Sato et al. 1993). El estado teliomorfo de Peridermium holwayi Syd., también se ha comunicado como parásito de la Teácea Wickstroemia sp. en Hawaii (Gardner 1992, 1994) y de Arctostaphylos uva-ursi ( Cash 1953, Cooke 1955, Anónimo 1960, Eglitis et al. 1966)

Exobasidium escalloniae Gómez \& Kisimova (Rev. Biol. Trop. 45: 1297. fig. 5, 1997).

Especie descrita de material costarricense como parásito en Escallonia myrtilloides L.f. var. patens (R. \& P.) Sleumer, se comunica aquí de material ecuatoriano y sobre el mismo hospedero. Las lesiones observadas son también hipertrofias fusiformes de talluelos, con alargamiento de entrenudos, alta concentración de antocianinas, un mayor tamaño de las hojas del eje parasitado pero que, sin embargo, no manifiestan infección. Los basidios aparecen en fascículos dispersos en la epidermis caulinar. Tanto el material costarricense como el ecuatoriano seca con una peculiar coloración azul pizarra.

Material estudiado: Ecuador: Matorrales andinos en quebrada, con abundancia de Espeletia hartwegiana, Diplostephium lavandulaefolium, Diplostephium sp., Stipa ichu, Festuca sp. y Puya clava-hercules, Páramo El Angel, Provincia Imbabura, 3150 m, Gómez \& Hewson 25967, 3/11/97 (QCNE, USJ).

Material costarricense adicional: Unos 5 km antes del cráter, Volcán Poás, Alajuela, 1500 m, I. Chacón \& L.D.Gómez 25979, 15/10/97 (USJ); 3 km sobre carretera al Irazú, San Juan de Chicuá, Prov. Cartago, 2800 m, 20/12/1997, L.D.Gómez \& I. Chacón 25998 (USJ).

Exobasidium jamaicense Gómez \& Kisimova, sp. nov. (= Exobasidium vaccinii s. Burt 1915, Gómez \& Kisimova, 1997).

Maculae bullatae roseo-lilacinae (sicco xerampelinae) folii Lyoniae formantes. Mycelium hypogenum hyphis numero-sis, intricatis, non fibulatis, pauce septatis, $1.8-2.1 \mu \mathrm{m}$ cras- sis, tenuis. Basidia 20 - $30 \times 4.7$ - $6 \mu \mathrm{m}$, 4-sterigma-tis, sporis ellipsoideis $14-15.2 \times 3-3.5$ $\mu m$, tenuis, hyalinis.

Holotypus: Sobre Lyonia jamaicensis (Swartz) D.Don. Jamaica, Cinchona, Schrenk s.n. (MO 44403). Material adicional: Jamaica. St. Andrew Parish. Cinchona, Hart 1280 (UCWI). St. Thomas Parish, near Sheldon, Harris 5208 (UCWI).

Hongo parásito hipofilo de Lyonia jamaicensis. Forma agallas buliformes en las hojas, cuando frescas de color rosado liláceo y cuando secan las agallas son café oscuro rojizo. Las hifas, intercelulares, sin fíbulas, con pocos septos, 1.8 - $2.1 \mu \mathrm{m}$ de diámetro, paredes delgadas, hialinas. Basidios 20 - 30 x 4.7 - $6 \mu \mathrm{m}$, esterigmas 4 , cortos $(2.5 \mu \mathrm{m})$, esporas elipsoidales, 14 - 15 x 3 - $3.5 \mu \mathrm{m}$, de paredes delgadas, hialinas.

La afinidad de la nueva especie es con $E$. emeritense Dennis del que difiere por las dimensiones de sus estructuras y el hospedero. En el cuarto fascículo de su monumental obra, Edward A. Burt (1915) inició un listado de las especies de Exobasidium, entonces consideradas como pertenecientes a Thelephoraceae. Burt fue el primero en publicar un registro de Exobasidium de la región neotropical al examinar el material recolectado por von Schrenk en Jamaica. Pero con un criterio diferente de la coespecificidad del basidiomiceto y sus hospederos, erróneamente asignó el material a Exobasidium vaccinii (Fkl.) Woronin, latissimo sensu Burt.

Exobasidium pernettyae Gómez \& Kisimova sp. nov. (Figs. 4,10 B).

Maculae supra rubrae infra albogrisaceae, planae rarissime pauciter bullatae, hypertrophiae nullae. Basidia 20.0 - $26.5 \times 4$ $4.5 \mu \mathrm{m}$, praecipue 4 -sporae. Basidiosporae hyalinae, tenuitunicatae, raro 2(3) - septate, $6.5-10.5 \times 2.0-2.5 \mu \mathrm{m}$. Conidia albido-translucidae $6.5-8.5 \times 1-2 \mu \mathrm{m}$.

Hongo parásito de Pernettya coriacea (Cav.) DC., hipógeno, himenio resupinado que forma una placa afelpada en el envés sin hiper- 


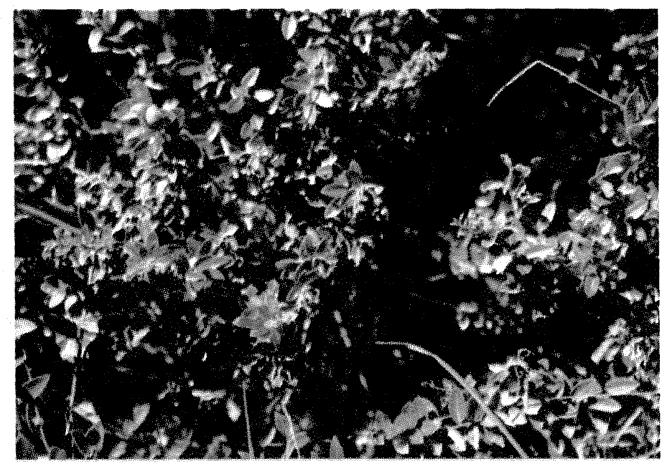

Fig. 4. Exobasidium pernettyae, parásito de Pernettya prostrata, holotipo. Foto: L. D. Gómez.

trofia de los tejidos foliares. En unos pocos casos se presenta una levísima deformación buliforme.

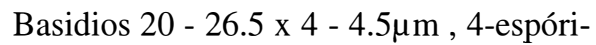
cos. Esporas hialinas, de paredes muy delgadas, raro (2)3- septadas, 6.5 - $10.5 \times 2.0-2.5$ $\mu \mathrm{m}$. Conidios translúcido-lechosos, bacilares, $6.5-8.5 \times 1-2 \mu \mathrm{m}$.

Holotipo: Costa Rica: Cráter Reventado, Macizo del Volcán Irazú, 3000 m, Provincia de Cartago, 20/12/1997, L. D. Gómez \& I. Chacón 25993A (USJ). Paratipo: loc. dat. cit. Gómez \& Chacón 25996 (USJ). Otros materiales: Sobre $P$. coriacea, $5 \mathrm{~km}$ al NE de San Juan de Chicuá, Cerro Nochebuena, 3000 m, Provincia de Cartago, 20/12/1997, L. D. Gómez \& I. Chacón 26000 (USJ); Villa Mills, 2900 m, Cartago, Agosto 1969, sobre P. coriacea, L. D. Gómez 3152 (CMI); 5 km NO de Carretera Interamericana Sur, Provincia San José, 3000 m, Agosto 1997, L. D. Gómez 25931 (USJ).

Inicialmente consideramos que el hongo parásito en Pernettya era una forma de E. vaccinii (Fkl.) Woronin y las últimas dos colecciones citadas arriba fueron incluidas como $E$. vaccinii en nuestra publicación anterior (Gómez \& Kisimova 1997). Sin embargo, el abundante material recolectado y observado en el macizo volcánico de Irazú, nos permite establecer que la $E$. pernettyae es predominantemente 4-espórica y las basidiosporas triseptadas en su mayoría, principal diferencia con $E$. vaccinii cuyas esporas son simples o uniseptadas, muy raramente biseptadas, los basidios son generalmente de $32-36 \times 7-8(10) \mu \mathrm{m}$, además del diferente hospedero.

\section{Exobasidium poasanum Gómez \& Kisi-} mova, nov. sp. (Fig. 5).

Maculae initialiter bullatae dein magnae planoconvexae, centrum albidum vel pallide roseum, a centro rubrum, foliis caulibusque Cavendishiae bracteatae parasitans. Fructificatio hypophylla, resupinata, coactata, albidogrisea, 45 - $72 \mu \mathrm{m}$ crassa, basidia $16-24.2 x$ 5 - $7 \mu \mathrm{m}, 2$-6-sterigmata, sporae ellipsoideae 12.5-2 $20.5 \times 3-4 \mu \mathrm{m}, 2$-septatae, crassitunicatae, hyalinae. Conidia cylindrica, $9-15 x$ $1.5-1.8 \mu \mathrm{m}$.

Holotypus: Costa Rica: En tallos y hojas tiernos de Cavendishia bracteata. Alrededores de Laguna Botos, Volcán Poás, Alajuela, 1600 m, I. Chacón \& L.D.Gómez 25981, 15/10/97 (USJ). Isotypus: loc. cit. (USJ).

Hongo parásito, endógeno, hipógeno en tallos y hojas de Cavendishia bracteata en que

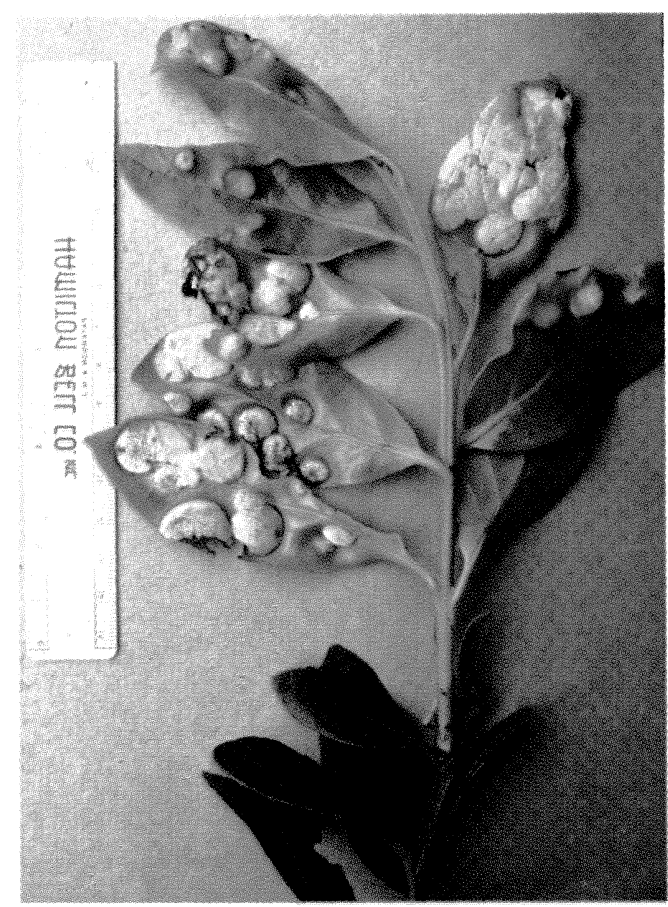

Fig. 5. Exobasidium poasanum. Agallas buliformes foliares e hipertrofia fusiforme del tallo en Cavendishia bracteata, holotipo, Volcán Poás, Costa Rica. Foto: L. D. Gómez. 
causa manchas foliares inicialmente buliformes que se tornan en enormes deformaciones planocóncavas, en el centro rosado pálido y en la periferia rojas, y en los tallos un abultamiento fusiforme con alta concentración de pigmentos rojos. El himenio es hipófilo, afelpado y ocupa toda la superficie in-fectada pero sólo aflora en las agallas foliares. Basidios $16-24.2$ × 5 - 7 $\mu \mathrm{m}$, con 2 - 4 - 6 (raro) esterigmas. Esporas elipsoidales, 12.5 - 20.5 x $3-4 \mu \mathrm{m}, 2$-septadas, de paredes gruesas, hialinas. Conidios cilíndricos, 9 - 15 x 1.5 - $1.8 \mu \mathrm{m}$.

Del grupo de especies de E. gaylussaciae P. Henn., y E. emeritense Dennis, difiere de esas especies por el número de esterigmas, las dimensiones de basidios y esporas, el tipo de lesión fusiforme del tallo y las agallas foliares y por el hospedero. Exobasidium flos-cavendishiae Kisimova \& Gómez tiene basidios, esporas y conidios diferentes y sólo infecta las brácteas florales de Cavendishia capitulata Donn.-Sm.

Exobasidium sphyrospermii Gómez \& Kisimova, nov. sp. (Fig. 6).

Gallae bulliformiae folii Sphyrospermo cordifolio Benth. formans. Mycelium endogenum, hypogenum. Systema hyphalis monomiticus, hyphis cylindraceis, tenuitunicatis, 1.5 - 2 $\mu \mathrm{m}$ crassis, pauce sed regulariter septatis. $\mathrm{Ba}$ sidia (20)21-27 x 2.5 -3(4) $\mu \mathrm{m}, 2$-sterigmata, sporae $6-10.5 \times 1$ - $2 \mu \mathrm{m}$, uniseptatae, tenuitu-

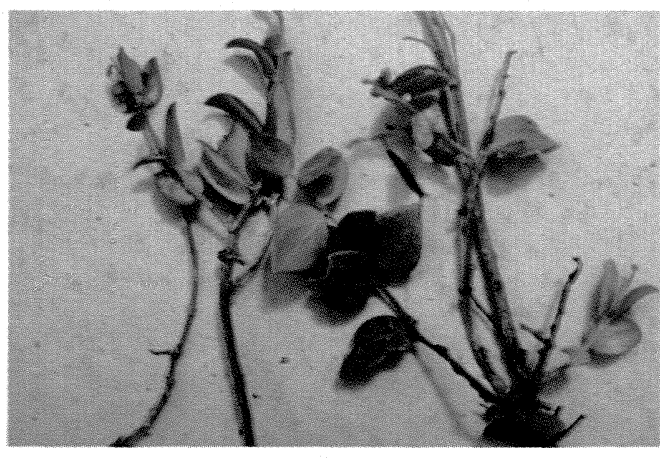

Fig. 6. Exobasidium sphyrospermii sobre Sphyrospermum cordifolium, holotipo. Talamanca, Costa Rica. Nótese la babosa y las escarificaciones en follaje infectado. Foto: L. D. Gómez. nicatae, hyalinae. Conidia suballan-toidea 9 $11 \times 1.5 \mu \mathrm{m}$.

Holotypus: Costa Rica: En bosque de Quercus copeyensis, Magnolia poasana, Araliaceae, Lauraceae, Melastomaceae. Parásito de Sphyrospermum cordifolium Bentham. Prov. Cartago, entre La Trinidad y Macho Gabb, km 62 Interamericana Sur, 2235 m. G. Hewson in Gómez 25956, 4/10/97 (USJ). Paratypus: loc. cit. Hewson \& Gómez 25957, 4/10/97 (USJ).

Parásito endógeno. Forma agallas buliformes en las hojas de Sphyrospermum cordifolium Benth., las lesiones de color rosado pálido. Micelio de hifas monomíticas, no fibuladas, cilíndricas, de paredes delgadas, hifas de 1.5 - $2 \mu \mathrm{m}$ de diámetro, con pocos septos regularmente distribuidos. Basidios (20)21 - $27 \mathrm{x}$ 2.5 - 3)4) $\mu \mathrm{m}, 2$-esterigmados, esporas de 6 10.5 x 1 - $2 \mu \mathrm{m}, 1$-septadas, de paredes delgadas, hialinas. Conidios subalantoideos $9-11 \mathrm{x}$ $1.5 \mu \mathrm{m}$.

Por sus características generales y las paredes delgadas de las esporas muestra cierta afinidad con E. vaccinii (Fkl.) Woronin y $E$. oxycocci Rostr., pero las dimensiones de los basidios y de los conidios son diferentes, particularmente las dimensiones de las esporas, que en $E$. vaccinii nunca son tan pequeñas. Del grupo de E. gaylussaciae P. Henn., que en su ámbito de distribución infecta especies de Gaultheria.

Exobasidium vaccinii (Fuckel) Woronin, Verh. naturf. Ges. Freiburg 4: 397. 1876.( Figs. 7, $10 \mathrm{~A})$.

Comunicado para Costa Rica por Gómez \& Kisimova (1977) se registra aquí para el Ecuador. Material estudiado: En V. floribundum H.B.K., Páramo El Angel, Prov. Imbabura, 3000 m, Gómez \& Hewson 25962 B y 25968, 3/11/97 (QCNE, USJ); Prov. Imbabura, en matorrales, sobre Vaccinium floribundum H.B.K., Cotacocha, 3200 m, Gómez \& Hewson 25965, 31/10/97 (QCNE, USJ). Material costarricense adicional: Camino a San Gerardo de Dota, 2700 m, Prov. San José, 19/12/1997, L. D. Gómez 25978 (USJ). 


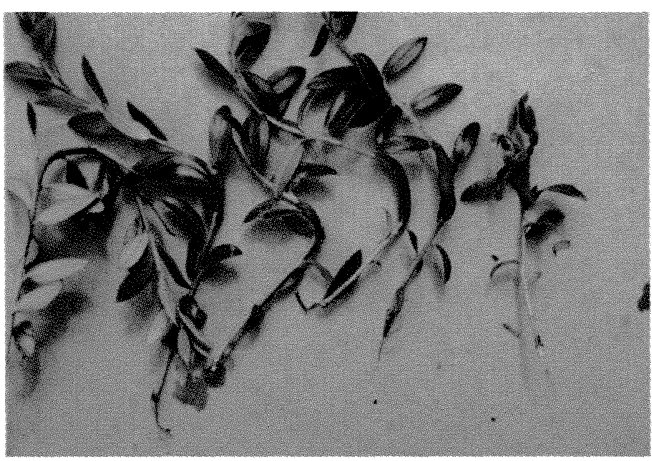

Fig. 7. Exobasidium vaccinii. Hipertrofias fusiformes típicas en tallos de Vaccinium consangineum. Foto: L. D. Gómez.

Las lesiones observadas en Ecuador son las típicas de la especie: hipertrofias fusiformes en tallos, agallas buliformes en hojas, infección total de los ápices de ramas serotinas, pero observamos una mayor incidencia de infección en frutos que la que se presenta en Costa Rica. Sin embargo, aunque las medidas de las estructuras reproductivas corresponden a la especie de Woronin, no puede descartarse la posibilidad de que la especie propuesta por Nickerson, E. perenne Nickers.,( Can. J. Plant Pathol. 6: 218 - 220, 1984), sea una entidad discreta y distinta de E. vaccinii Wor. y de $E$. oxycoccii Rostrup, a pesar de la tendencia de algunos fitopatólogos de reunir bajo el epíteto vaccinii, todas las lesiones parecidas que se presentan en especies de Vaccinium ( $c f$. Burt 1915, Gardner, 1985).

\section{Cryptobasidiaceae, Cryptobasidiales}

En la publicación anterior (Gómez \& Kisimova 1997) mencionamos el único representante de este grupo en Costa Rica, Clinoconidium bullatum Syd., de tres localidades muy próximas entre sí. Aquí agregamos los siguientes nuevos registros:

Clinoconidium bullatum Sydow, Ann. Mycol. 24: 283. 1926. (Fig. 11).

Con las características citadas en la descripción original, a lo que agregamos que las agallas foliares son, externamente globulares y sobresalen en ambas superficies foliares, con una disminución de clorofilas y aumento de pigmentos rojizos y amarillos en la haz. En su interior, las cavidades son sinuosas y las hifas, sub lente ambarinas en masa tienen una coloración pardo clara y afloran al interior de la agalla en fascículos dispersos. Los basidios 8 - 15 x 2 - $3 \mu \mathrm{m}$, (1)2 - 4-espóricos. Esporas elipsoidales o aovadas, lisas, ligeramente atenuadas en ambos extremos, raramente globosas, 8 - 14 x 4 - $8 \mu \mathrm{m}$, las globosas de 5 - $9 \mu \mathrm{m}$ de diámetro.

Material estudiado: Costa Rica: En hojas de Cinnamomum sp ( Phoebe s. auct.), Lauraceae, Reserva de Monteverde, Provincia de Puntarenas, W. Haber 12277 (USJ, MO).

En la prolija descripción específica y de la lesión que brinda von Sydow (loc. cit.), ese autor anota lo siguiente: "Forma parum diversa in foliis vivis Phoebes costaricense Mez \& Pitt" y aquí también se comunica la presencia de esporas de dos tipos y dimensiones diferentes.

Clinoconidium farinosum (P. Henn.) Patouillard, Bull. Soc. Mycol. France 14: 156. 1898. (Figs. 8, 11).

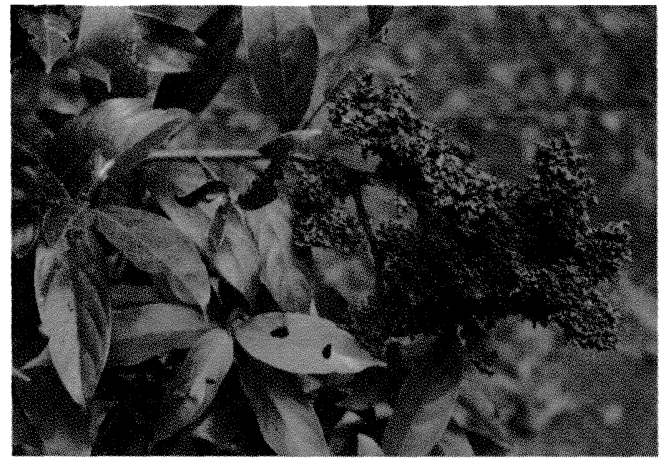

Fig. 8. Clinoconidium farinosum. Hipertrofias en yemas vegetativas de Ocotea monteverdensis. Foto: N. Wheelwright.

Hipertrofias de yemas vegetativas, infladas o tubulares, densamente escrofulosas, pardo rojizas, interiormente recubiertas por el micelio cuyas hifas afloran en fascículos densos, como acérvulos o esporodoquios, en toda la superficie interior que es sinuosa. Esporas en masa pardas, sub lente hialinas o ambarino muy pálido, diminutamente punteadas, 9 - $14 \times 8$ - $11 \mu \mathrm{m}$. Hifas conidióforas hialinas, filiformes, de hasta $50 \mu \mathrm{m}$ de largo. Conidios hialinos, ovoidales, 9 - $11 \times 8-10 \mu \mathrm{m}$. 


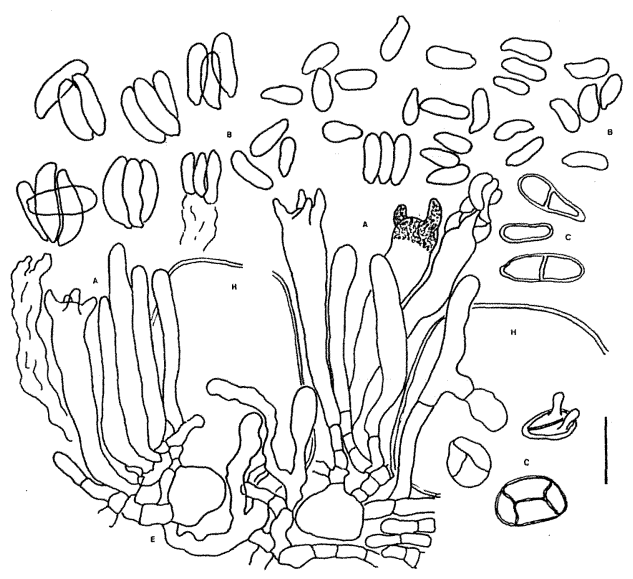

Fig. 9. Exobasidium arctostaphyli.a- probasidios y basidios, b- esporas, c- artrosporas, e- micelio intercelular, h- celulas epidérmicas del hospedero. Barra mide $10 \mathrm{~m}$.

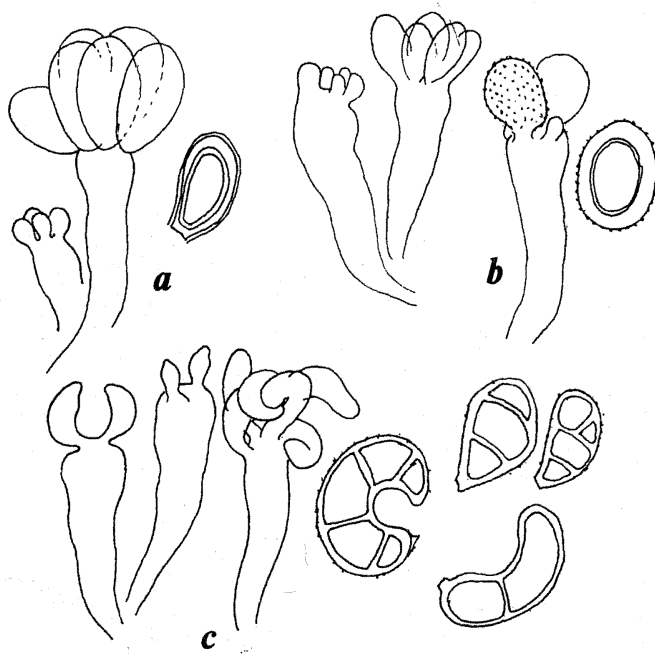

Fig. 11. Estructuras reproductivas de Cryptobasidiaceae costarricenses. a- basidios y esporas de Clinoconidium farinosum; b- basidios y esporas de Clinoconidium bullatum; c- basidios bipluriesterigmados y d- esporas de Drepanoconis larviformis.

Material estudiado: Costa Rica: En yemas foliares de Ocotea monteverdensis W. C. Burger, Lauraceae, Reserva de Monteverde, Puntarenas, Diciembre 1997, W. Haber 12290 (USJ, MO).

Esta especie se había comunicado antes de Puerto Rico (Stevenson 1975) en Nectandra $s p$., y de varias localidades sudamericanas en especies de Cryptocarya, Nectandra, Ocotea y

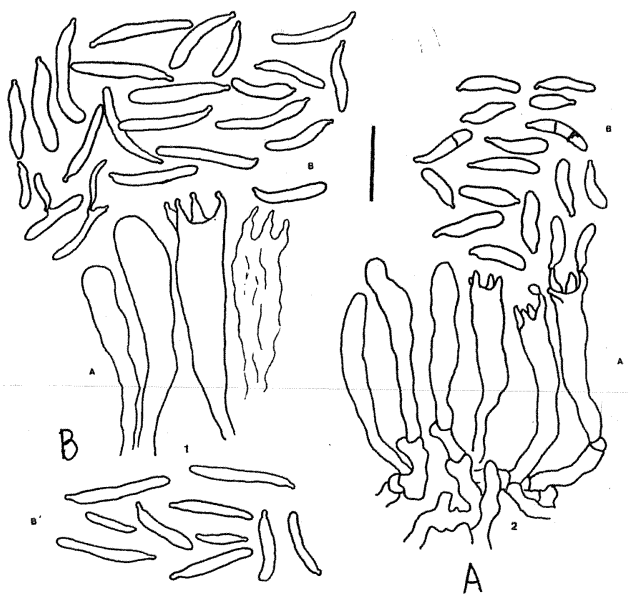

Fig. 10. A- Exobasidium vaccinii, basidios y esporas: BExobasidium pernettyae. a- probasidios y basidios, besporas. Barra mide $10 \mu \mathrm{m}$.

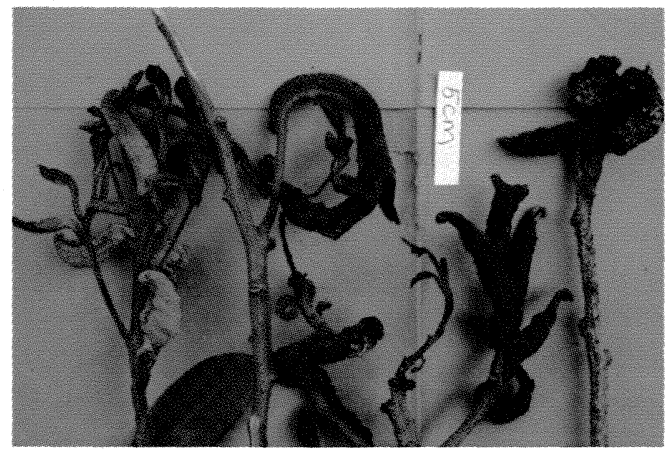

Fig. 12. Drepanoconis larviformis hipertrofia los tejidos de Aiouea costaricensis.

Oreodaphne ( Bitancourt \& Rossetti 1946, Viégas 1961). El material típico proviene de Brasil (Glaziou 22689) y este registro es el primero centroamericano, continental.

Drepanoconis larviformis (Speg.) Speg., Anales Museo Nacional de Buenos-Aires, ser. III, 2: 7. 1903; Fungi Guaranitici I: 158. 1884 pro Helicomyces (?) larviformis Spegazzini). (Figs. 11, 12, 13).

El género Drepanoconis fue erigido por Schroeter y Hennings en 1896 ( Beitr. zur Pilzflora Sudamerikas I, Hedwigia 33: 211) tipificado por D. brasiliensis para un parásito en Nectandra (Lauraceae). La detallada descripción de Schroeter \& Hennings permitió a Spegazzini 

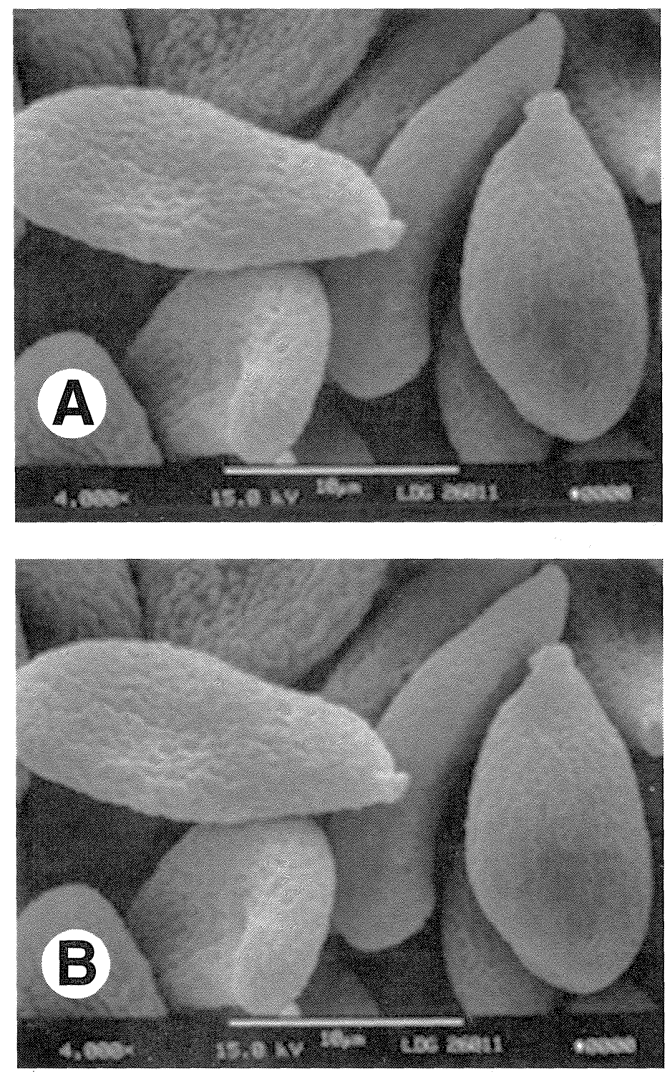

Fig. 13. A. Esporas de Drepanoconis Larviformis. B. Detalle, la barra mide $10 \mu \mathrm{m}$.

reconocer la sinonímia de Helicomyces larviformis con el hongo brasileño. El género ahora alberga seis especies, todas excepto una australiana, de lauráceas sudamericanas. Aquí se comunica Drepanoconis larviformis (Speg.) Speg. como parasito en Aiouea costaricensis (Mez) Kosterm.

Agallas en yemas foliares y caulinares, con alta deformación de los tejidos y en la madurez endurecidas, con superficie agrietada y rugosa. Basidios 40 - 50 × 3 - 4 $\mu \mathrm{m}$, Esporas en masa amarillo mostaza que al secar se torna opaco. Las esporas son de dimensiones muy variadas, 12 - $30 \times 6-14 \mu \mathrm{m}$ pero todas presentan paredes gruesas $(3-4.5 \mu \mathrm{m})$ y $1-4$ septos, de forma ovalada o angostamente piriformes o mazudas, con una cicatriz basal muy evidente.

Material estudiado: Costa Rica. En bosque nublado de San Gerardo de Dota, Prov. San José, 2700 m s.l.m., L. D. Gomez, G. Hewson \& N. Bynum en Gómez 26011 (USJ).

\section{OBSERVACIONES}

En muchas de las recolecciones en el campo, o durante el estudio de las muestras frescas en laboratorio, hemos observado la presencia de babosas adultas de la familia Limacidae. En nuestro primer artículo sobre Exobasidiales se indica en una de las fotografías las raspaduras y escarifi-caciones del himenio, en una agalla foliar, causadas por babosas. La presencia de babosas en las muestras costarricenses y de los altos páramos ecuatorianos nos hacen sospechar que esos moluscos, cuyas heces y limos podrían acarrear (y servir como adherentes) esporas viables, pueden ser vectores importantes de estos hongos parásitos (Voglino 1895).

Sin embargo, si la dispersión de esporas fuese por moluscos herbívoros, las llamativas hipertrofias de la mayoría de las infecciones no tendrían razón de ser en términos de economía fisiológica del binomio hospedero/parásito (Antonovics \& Miller 1989), de epidemiología de la infección o de la dinámica poblacional y genética de ambos componentes del sistema, como sucede con Caryophyllaceae parasitadas por Ustilago spp. (Antonovics \& Miller 1992, Miller, Antonovics \& Kelly 1993, Antonovics, Iwaza \& Hassell 1995, Miller et al 1996). Debe suponerse que otros vectores desempeñan un papel importante en la dispersión de los basidiomicetes (Ingold 1971, Jennersten 1983, Bultman \& White 1988, Roy 1994). El ciclo en el mantillo de los tallos y hojas infectados y de los estadios meióticos y conidiales en suelos donde germinan semillas de los hospederos, se desconoce, excepto por lo que informan Batra \& Batra (1985) sobre la hibernación en suelo de bayas contaminadas de Vaccinium, pero esos autores sugieren que los retoños primaverales son infectados por estadios sexuales acarreados por viento. Si las especies de Exobasidium dependieran del síndrome de anemocoria, la epidemiología de la infección sería mucho más generalizada. 
CONSIDERACIONES BIOGEOGRAFICAS: Una serie de apuntes sobre la distribución de Exobasidium y Cryptobasidiales con relación a la de sus plantas hospederas, se presenta con cierta extensión en Gómez \& Kisimova (1997). La exploración de los páramos y tierras altas de los Andes Ecuatorianos ha resultado en el descubrimiento de una nueva especie, $E$. aequatorianum, que muy probablemente se extienda al sistema andino de Colombia y del Perú, porque lo encontramos en toda la Cordillera del sur al norte del Ecuador asociado siempre con Vaccinium crenatum. El registro de $E$. escalloniae Gómez \& Kisimova en Escallonia myrtilloides en Ecuador indica que la coespecificidad del basidiomicete con su hospedero se mantiene en todo el ámbito geográfico de esa especie de Escallonia, un género de una cuarentena de especies que se distribuyen desde las tierras altas centroamericanas hasta Bolivia. E. vaccinii, descrito de Europa parece estar ampliamente distribuido en las zonas templadas y climas subtropicales, y representa sin duda un complejo de microespecies de difícil taxonomía por lo exiguo de sus características morfológicas. Nuestra hipótesis es que las cuatro nuevas especies que se describen de Costa Rica, E. disterigmicola, E. poasanum y $E$. sphyrospermii, E. pernettyae de Disterigma humboldtii, Cavendishia bracteata, Sphyrospermum cordifolium, y Pernettya prostrata, respectivamente, serán encontrados a lo largo y ancho de las distribuciones de esas ericáceas que abarcan desde Veracruz, Mexico, hacia el sur por Centro América hasta Bolivia y desde las montañas de Panamá-Colombia hacia el este hasta el Estado Sucre, Venezuela, en un ámbito altitudinal que fluctúa de 2000 a $4090 \mathrm{~m}$.

De particular interés resulta la nueva especie Exobasidium jamaicense parásito de Lyonia jamaicensis, de Jamaica. El género Lyonia se divide en cuatro secciones (Judd, 1995): 1- Pieridopsis, con 5 especies distribuidas en un arco desde el norte de Pakistán hacia el este hasta Japón y hacia el sur hasta Indonesia. 2-Arsenococcus, con una sola es- pecie del este de E.E.U.U., 3- Maria, con dos especies de la planicie costera del este de E.E.U.U., una de ellas, $L$. lucida, se extiende hasta Cuba e Isla de Pinos, 4- Lyonia, con 28 especies distribuidas en las Antillas excepto por dos de la planicie costera norteamericana y una de las montañas del oriente y sur de Mexico, con una distribución que se ajusta al concepto propuesto por Howard (1973) de "western continental distribution". La presencia de una especie de Exobasidium en el arco insular de las Antillas sugiere para este basidiomicete una paleogeografía neártica relativamente reciente. Ciferri (1961) cita, con duda, un registro de $E$. vaccinii, sin testigo y tal vez procedente de Haití. Un caso de particular interés es la presencia en las cadenas montañosas central y austral costarricenses de Exobasidium arctostaphyli, aparentemente un imigrante boreal junto con su hospedero Arctostaphylos s.l. y que desde Alaska hasta Guatemala es simpátrico con Abies Mill. (Pinaceae), este último hospedero del ecidio de Pucciniastrum goeppertianum, uredinal que parasita Vaccinium spp. Las demás especies centroamericanas y andinas de Exobasidium, posiblemente emigrantes desde poco antes del Mioceno, sugieren una distribución antártica, como indicamos en nuestro artículo anterior, para explicar la distribución circumpacífica de los parásitos y sus hospederos.

\section{AGRADECIMIENTOS}

A Gail Hewson por descubrir los primeros ejemplares de E. aequatorianum y E. sphyrospermii, y su paciente compañía en la exploración del Ecuador. A Isidro Chacón por su estimulante camaradería en las montañas costarricenses. Jorge Gómez-L. y James Luteyn identificaron las ericáceas hospederas. Nat Wheelwright facilitó la fotografía de Clinoconidium farinosum B. strack, Field Nuseum, Chicago, hizo la fotografia al MEB. A la Organización para Estudios Tropicales por facilitar mis investigaciones. 


\section{RESUMEN}

Se describen seis nuevas especies (Exobasidiales, Exobasidiaceae): Exobasidium aequatorianum parásito de Vaccinium crenatum (Don) Sleumer en los Andes ecuatorianos; Exobasidium jamaicense como parásito de Lyonia jamaicensis (Sw.)D.Don de Jamaica, Exobasidium disterigmicola como parásito de Disterigma huboldtii (Klz.) Nied., de Costa Rica y que forma distrofias del tipo escoba de bruja, Exobasidium sphyrospermii de Costa Rica, como parásito de Sphyrospermum cordifolium Bentham, Exobasidium poasanum, de Costa Rica y que forma gigantescas pseudoagallas en hojas y yemas de Cavendishia bracteata (R. \& P. ex St.-Hil.) Hoer., Exobasidium pernettyae, de Costa Rica y parásito foliar de Pernettya prostrata (Cav.) DC. Es muy posible que esas especies de parásitos se extiendan en todo el ámbito geografico de las plantas que parasitan, como lo demuestra el hallazgo de Exobasidium escalloniae Gómez \& Kisimova, descrito de Costa Rica, en Es. callonia myrtilloides var. patens, en Ecuador de donde tambien se registra Exobasidium vaccinii (Fkl.) Wor. Como parásito de las especies costarricenses de Arctostaphylos y Comarostaphylos, se registra, describe e lustra la especie Exobasidium arctostaphyli Hark., descrita de la costa occidental de los E.E.U.U. lo que constituye un nuevo registro neotropical. La exploración en búsqueda de criptobasidiáceas eleva el número de Cryptobasidiales registrados en Costa Rica a tres: Clinoconidium bullatum Sydow, originalmente descrito de las inmediaciones de Grecia, Alajuela, ahora se conoce también de Monteverde, Puntarenas, como parásito de Cinnamomum spp., Clinoconidium farinosum (P.Henn.) Pat., de la misma localidad y como parásito de Ocotea monteverdensis Burger. En la Cordillera de Talamanca, se localizó una población de Aiouea costaricensis (Mez) Kosterm., con numerosas agallas foliares y caulinares causadas por Drepanoconis larviformis (Speg.) Speg. Con excepción de $C$. bullatum, todos son nuevos registros centroamericanos.

\section{REFERENCIAS}

Anónimo. 1960. Index to plant diseases in the United States.U.S. Dept. Agriculture, Washington DC, Handb. $165,531 \mathrm{p}$.

Antonovics, J. \& H. E. Miller. 1989. pp. 185 - 214. The concept of fitness in plant-fungal pathogen systems. En K.J.Leonard \& W.E. Fry (eds). Plant disease epidemiology. McGraw-Hill, Nueva York, vol. 2.

Antonovics, J. \& H. Miller. 1992. Epidemiology of anthersmut infection of Silene alba $(=S$. latifolia $)$ caused by Ustilago violacea: patterns of spore deposition in experimental populations. proc. Royal Soc. London B, 250: 157 - 163 .
Antonovics, J., Y. Iwasa \& M. P. Hassell. 1995. A generalized model of parasitoid, venereal, and vector-based transmission processes. Am. Nat. 145: 661 - 675.

Batra, L.R. \& S.W.T. Batra. 1985. Floral mimicry induced by mummy-berry: Fungus exploits host's pollinators as vectors. Behav. Ecol. Sociobiol. 228: 1011 $-1013$.

Bitancourt, A. A. \& V. Rossetti. 1946. As galhas pulverulen tas das lauraceas. O' Biologico 12: 55 - 62.

Bultman, T.L. \& J. F. White. 1988. "Pollination" of a fungus by a fly. Oecologia (Berlin) 75_317 - 319 .

Burt, E. A. 1915. The Thelephoraceae of North America.IV. Exobasidium. Ann. Missouri Bot. Garden 2: $627-656$.

Cash, E. K. 1953. A checklist of Alaskan fungi. Pl. Dis. Reporter Suppl. 219: 1 - 70.

Ciferri, R. 1961. Mycoflora Domingensis Integrata. Ist. Bot.della Universitá di Pavia, 19, p. 65.

Cooke, W. B. 1955. Fungi of Mt. Shasta. Sydowia 9: 94 215 .

Eglitis, M, C. J Gould \& F. Johnson. 1966. Fungi found on Ericaceae in the Pacific coastal area. Washington State Agric. Exp. Sta. Bull. 675: 1 - 21.

Gardner,D. E. 1985. Red leaf disease of native Hawaiian species of Vaccinium spp., caused by Exobasidium vaccinii. Pl. Dis. 69: 805 - 806

Gardner, D.E. 1992. Hawaii's elusive akia rust. Hawaiian Bot. Soc. Newsl. 31: 44 - 46.

Gardner, D.E. 1994. The native rusts of Hawaii. Can. J. Bot. 72: 976 - 989.

Gómez P., L. D. \& L. Kisimova-Horovitz. 1997. Basidiomicetos de Costa Rica. Exobasidiales, Cryptobasidiales. Notas históricas, taxonómicas y fitogeográficas. Rev. Biol. Trop. 45: 1293 - 1310.

Hiratsuka, N. 1958. Revision of the taxonomy of Puccinias-traceae, with special reference to the species of the Japanese Archipelago. Kasai, Tokio, 167 p.

Howard, R.A. 1973. The vegetation of the Antilles, p. 1 38. In A. Graham (ed). Vegetation and veget ational history of northern Latin America. Elsevier, Nueva York. 393 p.

Ingold; C. T. 1971. Fungal spores: Their liberation and dispersal. Oxford University, Clarendon, 296 pp. 
Jennersten, O. 1983. Butterfly visitors as vectors of Ustilago violacea spores between Caryophyllaceous plants. Brittonia 40: 125 - 130.

Judd, W. S. 1995. Lyonia, Ericaceae.II. The Superior Ovaried genera. Flora Neotropica 66: 222 - 294.

Miller A., H., J. Antonovics \& A. W. Kelly. 1993. Genotypic variation in plant disease resistance - physicological resistance in relation to field disease transmission. J. Ecol. 81: 325 - 333.

Miller A, H., P. Thrall, J. Antonovics, A. Jarosz \& P. Oudemans. 1996. Population dynamics and genetics of plant disease: A case study of anther-smut disease. Ecology 77: 990 - 996.

Mulenko, W. 1994. Notes on some rare or unusual species of parasitic fungi. Mycologist 8: $71-75$.

Roy, B.A. 1994. The use and abuse of pollinators by fungi. Tr. Ecol. Evol. 9: 335 - 339.
Sato, S., K. Katsura \& Y. Hiratsuka. 1993. Morphology, taxonomy and nomenclature of Tsuga-Ericaceae rusts. Trans. Mycol. Soc. Japan 34: 47 62.

Savile, D. B. O. 1959. Notes on Exobasidium. Can. J. Bot. 37: $641-656$.

Shaw, C. G. 1973. Host fungus index for the Pacific Northwest.I. Hosts. Washington State Agric. Exp. Sta. Bull. 765: 1 - 212.

Stevenson, J. A. 1975. Fungi of Puerto Rico and the American Virgin Islands. Contr. Reed Herbarium 23. $743 \mathrm{p}$.

Viégas, A. P. 1961. Indice de fungos da America do Sul. Inst. Agronomico, Campinas, Brasil. 630 p.

Voglino, P. 1895. Richerche intorno all' azione della lumacha e dei rospi nello sviluppo di Agaricini. Nuovo Gior. Bot. Italiano (Forli) 27: 181 -185. 